\title{
CAPACIDADES MOTORAS ENVOLVIDAS NA HABILIDADE PSICOMOTORA DA TÊCNICA DE RESSUSCITAÇÃO CARDIOPULMONAR: SUBSÍDIOS PARA O PROCESSO ENSINO-APRENDIZAGEM
}

\author{
MOTOR CAPACITIES INVOLVED IN THE PSYCHOMOTOR SKILLS \\ OF THE CARDIOPULMONARY RESUSCITATION: \\ SUBSIDIES OF THE TEACHING-LEARNING PROCESS
}

\section{CAPACIDADES MOTORAS INVOLUCRADAS EN LA HABILIDAD PSICOMOTORA DE LA TÉCNICA DE RESUCITACIÓN CARDIOPULMONAR: SUBSÍDIOS PARA EL PROCESO ENSENANZA-APRENDIZAJE}

\begin{abstract}
Ana Maria Kazue Miyadahira*
Miyadahira AMK. Capacidades motoras envolvidas na habilidade psicomotora da técnica de ressuscitação cardiopulmonar: subsídios para o processo ensino-aprendizagem. Rev Esc Enferm USP 2001; 35(4): $366-73$.

RESUMO

Trata-se de uma estudo bibliográfico sobre a identificação das capacidades motoras envolvidas na habilidade psicomotora da técnica de ressuscitação cardiopulmonar (RCP) cuja finalidade é obter subsidios para o planejamento do processo ensino-aprendizagem desta habilidade. Verificou-se que as capacidades motoras envolvidas na habilidade psicomotora da técnica de RCP são predominantemente cognitivas e motoras, envolvendo 9 capacidades perceptivo-motoras e 8 capacidades de proficiência física. A técnica de RCP é uma habilidade psicomotora classificada como aberta, seriada e categorizada como uma habilidade fina e global e o processo de ensino-aprendizagem da técnica de RCP tem alto grau de complexidade.
\end{abstract}

PALAVRAS -CHAVE: Ensino. Ressuscitação cardiopulmonar. Aprendizagem.

\begin{abstract}
It is a bibliographic study about the identification of the motor capacities involved in the psychomotor skills of the cardiopulmonary resuscitation (CPR) which aims to obtain subsidies to the planning of the teaching-learning process of this skill. It was found that: the motor capacities involved in the psychomotor skill of the CPR technique are predominantly cognitive and motor, involving 9 perceptive-motor capacities and 8 physical proficiency capacities. The CPR technique is a psychomotor skill classified as open, done in series and categorizedas a thin and global skill and the teaching-learning process of the CPR technique has an elevated degree of complexity.
\end{abstract}

KEYWORDS: Teaching. Cardiopulmonary resuscitation. Learning.

\section{RESUMEN}

Se trata de un estudio bibliográfico sobre la identificación de las capacidades motoras involucradas en la habilidad psicomotora de la técnica de resucitación cardiopulmonar (RCP) cuya finalidad fue obtener subsidios para la planificación del proceso ensenanza-aprendizaje de esta habilidad. Se verifico que las capacidades motoras involucradas en la habilidad psicomotora de la técnica de RCP son predominantemente cognitivas y motoras, involucrando 9 capacidades perceptivomotoras y 8 capacidades de proficiencia física. La técnica de RCP es una habilidad psicomotora clasificada como abierta, seriada y categorizada como una habilidad fina y global y el proceso de ensenanza-aprendizaje de la técnica de RCP tiene alto grado de complejidad.

PALABRAS-CLAVE: Enseñanza. Resucitación cardiopulmonar. Aprendizaje.

Enfermeira- Prof. Titular do Departamento de Enfermagem Médico-cirúrgica da EEUSP.. 


\section{INTRODUÇÃO}

A prática da enfermagem pressupõe competência em uma série de habilidades predominantemente psicomotoras"(1-16). Essas habilidades compreendem desde as mais simples atividades até as mais complexas, as quais envolvem grande número de movimentos coordenados e de alta precisão. Isso explica a preocupação dos professores na área profissionalizante, relacionada com o processo ensinoaprendizagem das habilidades psicomotoras.

Considerando que a habilidade psicomotora é um dos instrumentos básicos da enfermagem, concluise que é indispensável seu desenvolvimento como um meio de trabalho e não como um fim (17-18)

O desenvolvimento da habilidade psicomotora específica se faz por meio do ensino das técnicas/ procedimentos de enfermagem. Em vista disso, as técnicas/procedimentos de enfermagem que exigem habilidade psicomotora, no processo ensinoaprendizagem são ministrados, de maneira gradativa, em várias etapas, segundo o grau de complexidade para facilitar a aprendizagem.

Embora praticamente não existam pesquisas demonstrando como ocorre a organização do movimento e seu efeito sobre o desempenho de uma habilidade psicomotora, alguns autores (19-25) citam que, além desta aprendizagem por etapas, é fundamental, ainda, a prática repetida que permite ao estudante atingir o desempenho desejado, segundo o sistema de retroalimentação imediata e precisa.

Atualmente, aceita-se que o conceito de "feedback" ou conhecimento do resultado, no processo ensino-aprendizagem das habilidades psicomotoras não se restrinja ao conhecimento dos erros, mas sim tomado no seu significado geral de qualquer tipo de informação sensorial sobre o movimento, não exatamente com referência a erros (26). O "feedback" na aprendizagem motora tem três funções principais. Primeira: é uma fonte de informação para ajudar o estudante ou aprendiz a ajustar sua resposta para a próxima tentativa; segunda: funciona como um reforço quando uma tentativa está total ou parcialmente correta e finalmente é um meio de motivação, pois permite ao estudante ter a informação de como está progredindo em relação ao objetivo do desempenho estabelecido(22).

Um fator relevante a ser considerado é que as pessoas diferem na capacidade de aprender habilidades psicomotoras, pois dependem das capacidades motoras presentes. Estas diferenças são devidas a fatores genéticos ou podem ser atribuídas à totalidade de experiências anteriores de cada estudante? Uma vez presentes, como este conhecimento é adquirido?

Alguns autores(15-16,27-33) citam que as áreas relacionadas com os procedimentos e as técnicas de enfermagem ainda não foram suficientemente abordadas e salientam a necessidade de estudos.

Este estudo pretende identificar na literatura as capacidades motoras envolvidas na habilidade psicomotora da técnica de ressuscitação cardiopulmonar (ROP), importantes no planejamento do processo de ensino-aprendizagem. Após um levantamento na literatura a respeito do assunto, obtivemos:

\section{Capacidades motoras e cognitivas envolvidas na técnica de ressuscitação cardiopulmonar (RCP).}

Para discutir-se o processo ensino-aprendizagem da técnica da ROP, é fundamental destacar que a técnica de ressuscitação cardiopulmonar é um procedimento predominantemente de domínio motor e, sendo assim, enfocar-se-ão os processos que servem de base para aprendizagem de capacidades cognitivas e motoras que conduzem à performance dessa atividade ou habilidade psicomotora específica. $\mathrm{Na}$ bibliografia a respeito do assunto(15-16,31-33,35-39), os termos movimento e motor são utilizados como sinônimos, mas para aqueles que fazem distinção entre ambos, define como movimento qualquer ação externa observável e como motor, os impulsos internos eferentes (40). Sustenta ainda que se o movimento for considerado uma criação que ocorre no espaço, pode-se classificá-lo como movimento não locomotor (resposta observável executada pelo corpo em uma posição estacionária, tal como bater palmas) ou como movimento locomotor (resposta observável executada pelo corpo, movimentando-se no espaço, de um ponto para outra, tal como um salto).

O termo capacidade é definido como um traço geral ou qualidade de um individuo relacionado com o desempenho de uma variedade de habilidades motoras, constituindo um componente da estrutura dessas habilidades. São traços ou qualidades inatos, relativamente permanentes, que na pessoa adulta são mais dificeis de se alterar ${ }^{(41)}$. Entretanto, esclarece que, embora a maioria dos teóricos do comportamento defendam que estas capacidades são geneticamente determinadas, existem poucas evidências empíricas desta conclusão, sendo o assunto polêmico (37). Assim, julgase prudente admitir que estas capacidades podem ser produtos da aprendizagem, pelo treinamento (prática) que se desenvolve em diferentes velocidades, principalmente durante a infância e a adolescência. Algumas capacidades dependem mais de fatores genéticos do que de fatores de aprendizagem, mas a maioria das capacidades depende de ambos os fatores em algum grau. Num determinado estágio da vida, constituem traços ou qualidades que o indivíduo traz consigo quando começa a aprender uma nova tarefa ou atividade.

O termo habilidade é definido pelo mesmo autor como ato ou tarefa que requer movimento e deve 
ser "adquirido ou aprendido" a fim de que a tarefa seja executada corretamente. Oonseqüentemente, é utilizado com referência ao nivel de proficiência na execução de uma tarefa específica ou de um grupo limitado de tarefas. Habilidade é então entendida como um indicador de qualidade de desempenho, qualidade essa geralmente julgada com base na produtividade do executante ou em certas características do seu desempenho. Desta forma, na técnica da ressuscitação cardiopulmonar, será considerado o termo habilidade ou desempenho o executar de forma completa e correta os itens que a compõem. A suposição é de que na execução de uma tarefa complexa seja fundamental a identificação das capacidades subjacentes.

Aponta ainda a importância de: primeiro, detectar as capacidades envolvidas em determinada habilidade; segundo, identificar a origem dos problemas ou dificuldades no desempenho dos estudantes e terceiro, prever o potencial individual do estudante no desempenho de tarefa específica(37). Esta definição pode ser ampliada considerando não apenas a proficiência na execução, isto é, a maximização da precisão, mas também a minimização do gasto energético físico, mental e do tempo utilizado no desempenho de uma tarefa (26). Na técnica de ressuscitação cardiopulmonar, em especial, estes aspectos são importantes pela situação específica em que a mesma é aplicada, isto é, em situação de emergência e no local da ocorrência.

Desta maneira, o nível de desempenho que um estudante pode adquirir, na execução de uma determinada habilidade psicomotora, dependerá das capacidades básicas individuais e do seu treinamento que será a prática dessa habilidade. Assim, quando se fala em aprendizagem de uma habilidade, como da técnica da ressuscitação cardiopulmonar, pressupõese a existência de uma série de capacidades básicas envolvidas em tal atividade.

Em 1972, Fleishman (42), tendo como base os resultados de algumas pesquisas (41,43), efetuou o desenvolvimento de uma "taxionomia" das capacidades perceptivo-motoras humanas. Neste estudo, o autor identificou e mensurou onze capacidades perceptivomotoras e nove capacidades de proficiência fisica.

Assim, tendo essa "taxionomia" como referencial teórico para análise, na habilidade psicomotora da técnica de ressuscitação cardiopulmonar as 9 capacidades perceptivomotoras identificadas foram: 1. Coordenação multimembro - capacidade de coordenar o movimento dos membros superiores e inferiores simultaneamente (Exemplo-"estimular a vítima balançando seu ombro"); 2. Precisão de controle - a capacidade de executar ajustes musculares altamente controlados e precisos em que grupos maiores de músculos estão envolvidos (Exemplo- "abrir as vias aéreas por meio da manobra de deslocamento do queixo nos casos de trauma"); 3. Orientação da resposta - capacidade de selecionar rapidamente quando uma resposta deve ser dada, como na situação de tempo de reação na qual uma escolha deve ser feita (Exemplo- "observar o tórax, com a face acima da boca da vítima, olhando em direção aos pés para ver, ouvir e sentir a respiração"); 4. Tempo de reação - capacidade de responder rapidamente a um estímulo quando ele aparece (Exemplo- "na ausência do pulso dizer: não tem pulso, ligue para 193, não precisa de ficha"); 5. Velocidade do movimento do braço capacidade de fazer um movimento geral e rápido do braço (Exemplo- "fazer quinze compressões, alternando com duas ventilações por ciclo"); 6. Controle de graduação - capacidade de mudar a velocidade e a direção de respostas no tempo adequado (Exemplo- "fazer massagem cardíaca externa, comprimindo o tórax, através de movimentos equivalentes a 3,5-5,0 cm de amplitude, por 15 vezes, no adulto"); 7. Destreza manual - capacidade de fazer movimentos hábeis de braço e mão, bem direcionados (Exemplo- "localizar o ponto de massagem no tórax, percorrer com uma das mãos o rebordo costal, marcar com o dedo anular o apêndice xifóide e colocar os dedos médio e indicador justapostos ao anular"); 8. Estabilidade braço-mão capacidade de fazer movimentos precisos de posicionamento de braço e mão, nos quais a força e a velocidade têm um envolvimento mínimo (Exemplo-"permitir o relaxamento do tórax, após cada compressão"); 9. Velocidade punho-dedos capacidade de mover o punho e os dedos com rapidez (Exempla-"após cinco ciclos, avaliar o retorno dos batimentos cardíacos").

As capacidades de proficiência fisica diferem das perceptivo-motoras no sentido de -que elas são freqüentemente relacionadas com o desempenho atlético e físico em geral, são portanto, as capacidades da condição física ("physical fitness"). As 8 capacidades de proficiência física identificadas na habilidade psicomotora em tela foram: 1. Força estática - máximo de força que pode ser exercido contra objetos externos (Exemplo- "manter as vias aéreas permeáveis"); 2. Força dinâmica - resistência muscular ao exercer força, repetidas vezes (Exemplo" fazer quinze compressões, alternando com duas ventilações por ciclo"); 3. Força de tronco - força dos músculos do tronco (Exemplo- " posicionar a vítima de costas, em bloco, imobilizando a cabeça e a região cervical"); 4. Flexibilidade de estiramento - capacidade de flexionar ou estender os músculos do tronco e das costas (Exemplo- "chamar em voz alta, perto do ouvido -Oomo está?"); 5. Flexibilidade dinâmica - capacidade de fazer flexões do tronco, repetidas e rápidas, como em uma série (Exemplo "contar em voz alta: $1,2,3, \ldots 15-80-100$ compressões/ minuto ou 15 compressões por 9-11segundos") ; 6. Coordenação geral do corpo - capacidade de coordenar a ação de várias partes do corpo, enquanto ele está em movimento (Exemplo- "posicionar corretamente os braços - ombro do socorrista deve 
estar na linha do esterno da vítima"); 7. Equilibrio geral do corpo - capacidade de manter o equilíbrio (Exemplo- "ajoelhar-se próximo ao ombro da vítima, mantendo as pernas afastadas - um joelho ao nivel da cabeça e o outro ao nível superior do tórax"); 8. Resistência cardiovascular - capacidade de sustentar um esforço máximo que requer esforço cardiovascular (Exemplo- "fazer quinze compressões, alternando com duas ventilações por ciclo").

Os três elementos fundamentais para quase todas as habilidades são: a) perceber os aspectos relevantes do meio ambiente; b) decidir o que fazer, onde e quando; c-) produzir atividade muscular organizada $^{(26)}$.

O autor considera ainda que os três componentes envolvidos na produção de muitas habilidades são:

1) os posturais, os quais ele denominou de "plataforma" para sustentar as ações, por exemplo: na habilidade psicomotora da técnica de ressuscitação cardiopulmonar, o socorrista tem que ter as capacidades perceptivo-motoras e de proficiência física, já citadas, para fazer as manobras desta técnica de forma efetiva;

2) os componentes de locomoção do corpo e dos membros até a ação, isto é, no exemplo da habilidade em estudo, a locomoção do corpo, movimentando-se em direção à vítima, o movimento de ajoelhar-se para fazer as manobras de ressuscitação cardiopulmonar:

3) os componentes de manipulação que produzem a ação, como por exemplo, os movimentos de coordenação e prontidão motora, flexibilidade dinâmica do tronco e membros, de forma repetida e rápida, na execução da massagem cardíaca.

Existem vários sistemas de classificação da habilidade em categorias, o que possibilita considerar as habilidades psicomotoras em termos do que elas têm em comum, sem se deter nas diferenças. Estas generalizações são importantes na aplicação de conceitos quando se trata do processo de ensinoaprendizagem dessas habilidades.

As habilidades podem ser classificadas em: abertas ou fechadas; discretas, contínuas e seriadas e, predominantemente, cognitivas ou motoras (26). Já MAGILL ${ }^{37)}$ faz a seguinte classificação: precisão do movimento; caráter bem definido dos pontos iniciais e finais e estabilidade do ambiente.

Frente a estes dois sistemas de classificação, a habilidade psicomotora da técnica de ressuscitação cardiopulmonar será considerada:

- Uma habilidade aberta, em que o ambiente é variável e imprevisivel durante a ação, e isto requer do socorrista uma resposta rápida às variações, de acordo com as condições vitais da vítima. Esta resposta resulta dos processos associados à percepção, padrão de reconhecimento e tomada de decisão. Essa é a classificação que Poulton, em 1957, denominou de estabilidade do ambiente. Esse sistema de classificação foi expandido para torná-la aplicável à instrução de habilidades esportivas, utilizando o termo tarefa externamente compassada ou forçada, em que o início da ação é determinada por uma fonte externa, o estímulo (44). Nesta pesquisa, o estímulo é a vitima. De acordo com a sua resposta, as condutas deverão ser tomadas. Assim, as condições da vítima orientarão as ações.

- Uma habilidade seriada ou de caráter bem definido dos pontos iniciais e finais de habilidades seriais, pois cada item constitui uma habilidade discreta, isto é, tem um início e um fim definidos, porém o conjunto dos itens do "check-list" constitui uma habilidade psicomotora nova, complexa quanto ao procedimento como um todo, em que cada item deve ser executado em uma ordem específica: ele é um estímulo e uma resposta, isto é, é uma resposta à conduta imediatamente anterior e um estímulo para a conduta seguinte. Assim, ao identificar a ausência do pulso, iniciar imediatamente a compressão cardíaca externa.

- Uma habilidade perceptivo-motora ou psicomotora, pois envolve não só a seleção do que fazer, mas como fazê-la. Na habilidade em pauta, assim como na maioria das habilidades reais, estas dimensões são contínuas porque não há habilidade completamente cognitiva ou habilidade totalmente motora. Toda habilidade, por mais cognitiva que possa parecer, exige pelo menos uma resposta motora, e toda habilidade motora exige alguma tomada de decisão. Assim, qualquer item da técnica de ROP exige a combinação de fatores perceptivos e motores com fatores de controle motor.

\begin{abstract}
- Uma habilidade em que as categorias não podem ser consideradas mutuamente exclusivas, como globais ou finas, pois requer uma coordenação perfeita e uma precisão de movimento. Alguns autores(19-22) classificaram a habilidade motora como fina ou global conforme o tamanho do músculo envolvido, a quantidade de força aplicada e a amplitude do movimento. Oonsiderando estes componentes para categorizá-las, a habilidade em tela envolve não só a grande musculatura como base principal do movimento, como os pequenos músculos; a quantidade de força aplicada é grande e a amplitude do movimento pode ser grande.
\end{abstract}

\section{Processo ensino-aprendizagem da habilidade psicomotora da técnica de ressuscitação cardiopulmonar.}

A aprendizagem é um fenômeno não observável diretamente; só pode ser inferida da mudança do comportamento ou do desempenho ou performance de 
uma pessoa. O desempenho, isto é, o comportamento observável, é o indicador chave que nos permite verificar se houve aprendizagem ou aquisição de uma habilidade. Julga-se ainda que a aprendizagem envolve um conjunto de processo no sistema nervoso central (SNO), que permite o desenvolvimento das capacidades adquiridas para um desempenho ou uma performance habilidosa.

Cabe lembrar que o processo de aprendizagem é bastante complexo, e que as estratégias de ensino são de grande relevância para professores que tendem a fazer uma abordagem moderna acerca desse processo, que são: diferenças individuais, motivação, concentração, reação (ansiedade), retroalimentação, memorização e retenção(45).

Quando se trata de avaliação da aprendizagem em situações de prática, Schimdt (26) lembra que a prática pode exercer dois tipos diferentes de influência no desempenho: um relativamente permanente, quando ocorre efetivamente a aprendizagem, e outro, somente temporário e transitório que tende a desaparecer com o tempo ou com uma mudança nas condições. Entre os vários fatores que podem interferir no desempenho, durante a prática, estão: a motivação, a fadiga, o uso de medicamentos, drogas, o ambiente de ensino. Com vistas a uma maior eficácia nas estratégias instrucionais de uma habilidade motora, é fundamental a compreensão dos estágios ou fases desta aprendizagem. Como sabemos, o processo ensinoaprendizagem envolve tempo e prática. Assim, um estudante para passar do papel de novato ou principiante para um desempenho habilidoso passa por vários estágios distintos que podem ser identificados no processo de ensino-aprendizagem.

Fitts, Posner (46) propuseram três estágios de aprendizagem, dependendo de como se processam as respostas necessárias.

O primeiro, o estágio cognitivo, caracterizase por envolver uma intensa atividade mental ou intelectual, à medida que o estudante procura por respostas relativas às próprias técnicas ou estratégias. Nesta fase de aprendizagem da habilidade psicomotora (RCP), de avaliar e prestar o atendimento primário em primeiros socorros, observa-se, comumente, preocupação de como começar, qual postura corporal correta, qual a seqüência dos itens do modelo padrão, como posicionar as mãos para manter as vias aéreas pérvias, como fazer para ocluir a narina e fazer a ventilação "boca a boca", como achar o ponto correto da massagem cardíaca. Este estágio, caracteriza-se por apresentar elevada quantidade de erros no desempenho, ser executada de forma irregular, incerta e descompassada, muitas vezes, com o ambiente. Embora o estudante identifique o erro na execução, geralmente desconhece a maneira de melhorar o seu desempenho.
O segundo, o estágio associativo, caracteriza-se pela mudança da natureza da atividade cognitiva. Os erros são menos freqüentes e menos grosseiros em sua natureza. O estudante está refinando na habilidade, pois consegue detectar alguns de seus erros e começa a reconhecer a forma de melhorar o seu desempenho. Assim, na habilidade em estudo, verifica-se que ele já sabe mentalmente a seqüência dos itens da técnica de ROP, tem postura e controle corporal consistente, consegue fazer a ventilação "boca a boca", mantendo as vias aéreas pérvias, consegue fazer a massagem cardíaca no ponto correto. A consistência cresce gradualmente, ainda, e o movimento começa a ser dominado e estável. A melhora da eficiência do movimento tende a reduzir o gasto energético.

O terceiro, o estágio autônomo, caracterizase por ser o estágio final da aprendizagem, em que a habilidade torna-se quase automática ou habitual. $\mathrm{O}$ estudante não necessita de atenção e de concentração ao desempenhar a habilidade. Esse estágio é o resultado de uma quantidade enorme de prática (treinamento), que permite ao estudante executar a ROP sem se concentrar exclusivamente no movimento, estando, portanto, em condições de dar atenção a outros aspectos da técnica que lhe permitirão um desempenho melhor. Na habilidade em pauta, o estudante consegue atender melhor aos aspectos cognitivos, psicomotores e afetivos que envolvem tal técnica. Este fato por ser comparado ao que SINGER (47) postulou "hierarquia de hábitos". Segundo esse autor, na aprendizagem de uma tarefa complexa, o estudante apreende, primeiro, as fases "fundamentais" da habilidade e, depois, começa a se concentrar nos aspectos mais avançados dessa habilidade. A autoconfiança aumenta e a capacidade de detectar seus próprios erros e de pensar em estratégias de como corrigi-los torna-se mais desenvolvida.

Adams, citado por Magill(22), em 1971, propôs dois estágios: o primeiro, estágio verbal motor, que incorpora o estágio cognitivo e associativo e o segundo, o estágio motor, que corresponde ao estágio autônomo de Fitts e Posner (46).

Já no modelo de Gentil (44), são descritos dois estágios:

O primeiro, o estágio designado de obtendo a idéia do movimento, caracteriza-se por requerer do estudante que identifique não só os estímulos relevantes e os não relevantes bem como o padrão de movimento mais adequado para um bom desempenho naquela habilidade. É durante este estágio de aprendizagem que o estudante se concentra em desenvolver a coordenação, praticando a habilidade de forma que o padrão de movimento obtido como resposta a esta prática seja o ideal ou característico desta habilidade. $\mathrm{Na}$ habilidade do procedimento em tela, julga-se que, nesse estágio, o estudante seja capaz 
de identificar qual a conduta a se tomada de acordo com a relevância do estímulo. Assim, se o estímulo é a condição da vítima e ela está com "pulso ausente", o estudante deve ter a prontidão motora de iniciar imediatamente as manobras de ressuscitação cardiopulmonar, ao invés de se preocupar em estancar a hemorragia, que é menos relevante na situação.

$$
\text { O segundo, o estágio de fixação/ }
$$

diversificação, caracteriza-se por requerer do estudante que ele se concentre na habilidade, de forma que ele obtenha um bom desempenho. Este estágio está relacionado com o tipo de classificação da habilidade. Assim, na habilidade classificada como fechada, o estágio será de fixação, isto é, como não há variação do ambiente, a prática permitirá ao estudante refinar o padrão de movimento aprendido no primeiro estágio, permitindo que seja capaz de produzir tal padrão de resposta sempre de forma correta ou eficaz. Já na habilidade classificada como aberta, que é o caso da habilidade deste estudo, o estágio será de diversificação, pois como o ambiente é variável e imprevisivel, o mesmo padrão de movimento não vai ser adquirido em respostas sucessivas. O foco desse estágio é desenvolver o estudante de forma que ele consiga se adaptar à criação do ambiente, com sucesso, isto é, tendo um desempenho, uma boa adaptação mesmo com as mudanças do ambiente. Acredita-se que o estudante seja capaz de obter bom desempenho frente à diversificação de padrões de movimentos, desde que no primeiro estágio tenha desenvolvido um largo repertório desses padrões, que vão dar as bases para a adaptação de variação do ambiente. Assim, no nosso exemplo, ele deve ter, já no primeiro estágio, desenvolvidos os padrões de movimentos, que lhe permitam que, mesmo se a condição do paciente, isto é, o estímulo mude, ele saiba fazer as manobras de ressuscitação cardiopulmonar ou estancar a hemorragia, por exemplo.

A identificação destes estágios de aprendizagem de uma habilidade motora é importante, pois fornece subsídios para que o professor utilize estratégias instrumentais adequadas àquele estágio de aprendizagem, permitindo que o estudante desenvolva o máximo de desempenho em cada estágio.

O centro do comando do comportamento humano é o sistema nervoso central (SNC). Este sistema é composto por duas estruturas, o cérebro e a medula espinhal, que formam a base do sistema de controle, que é o centro de atividade na integração e organização da informação sensorial e motora no controle do movimento. A produção de um movimento coordenado envolve: recepção sensorial da informação do ambiente que provém de uma variedade de fontes (músculos voluntários estriados e esqueléticos e sistemas visual e auditivo); transmissão eferente da informação relativa ao movimento a ser produzido e, nesta fase, são de importância fundamental os proprioceptores (fusos neuromusculares e as terminações neurotendíneas) e a integração da informação sensorial e motora na produção de um movimento, sendo que a transmissão eferente da informação de movimento do córtex caminha para a musculatura através dos sistemas piramidal e extrapiramidal (22)

Autores (22'48) descrevem que o sistema piramidal é o responsável pela transmissão eferente da informação que controla os movimentos voluntários finos (precisos) e o sistema extrapiramidal está relacionado com o refinamento ou com o ato de "suavizar" (modulação tônica - sinergia) os movimentos musculares iniciados pelo sistema piramidal. Também está envolvido na grande parte da informação do movimento para a musculatura referente a movimentos reflexivos grosseirs e posturais. Porém, acrescenta que o sistema extrapiramidal não é bem entendido hoje em dia por ser um sistema muito complexo.

Há os que consideram errôneo usar os termos piramidal e extrapiramidal, uma vez que o sistema piramidal foi assim denominado porque as fibras do feixe corticoespinhal lateral formam as pirâmides no bulbo (vias corticoespinhais). O sistema extrapiramidal é formado pelo restante do tronco cerebral descendente e as vias espinhais que não passam pelas pirâmides e estão relacionadas com controle postural. Entretanto, a via corticoespinhal ventral não passa pela pirâmides e várias fibras piramidais desempenham outras funções, e o sistema que é chamado extrapiramidal é constituído por várias vias distintas com múltiplas funções (49). Lembra, ainda, que os mecanismos de regulação da postura são múltiplos. Envolvem toda uma série de núcleos e várias estruturas, incluindo a medula espinhal, o tronco e o córtex cerebral. Eles estão relacionados não apenas com a postura estática, mas também, em conjunto com os sistemas corticoespinhal e corticobulbar, com o início e o controle do movimento. Para a técnica de ressuscitação cardiopulmonar, ora em estudo, assumiremos o conceito apresentado por alguns autores $(22,37,48,50)$ de que o sistema extrapiramidal está envolvido nos movimentos automáticos secundários mais complexos, que requerem automatismos de aquisição mais intelectualizados, adquiridos por um aprendizado consciente. Estes passam inicialmente por uma fase de execução voluntária, indo incorporar-se aos automatismos, propriamente ditos, somente quando se encontram perfeitamente organizados. Podemos comparar com o ato de dirigir um automóvel, que nada mais representa do que um ato práxico. Conseqüentemente, dada a participação voluntária desta categoria de movimento, o córtex cerebral é essencial para a sua execução.

Em 1970, propôs-se uma hipótese de interação entre o cerebelo e o córtex cerebral em termos de 
controle de movimento ${ }^{(51)}$. Nesta hipótese o córtex cerebral é responsável por comandos de início da resposta para o movimento voluntário "inexperiente", isto é, o primeiro estágio de aprendizagem na aquisição de habilidades. Em conseqüência da experiência, o local de controle desses movimentos se transfere para o cerebelo. Assim, à medida que o estudante progride no estágio inicial cognitivo de aprendizagem ao estágio final ou autônomo, o centro do controle do movimento se desloca do córtex cerebral para o cerebelo. Esta proposta parece consistente com os estágios sugeridos, na medida em que o tipo de atividade envolvida em cada estágio é considerada. Uma grande quantidade de atividade verbal ou cognitiva é necessária ao estágio inicial da aprendizagem, daí a necessidade do envolvimento do córtex cerebral. Mas, à medida que a automação do movimento resulta da prática, o papel da córtex cerebral se tornaria menos importante. As trajetórias de estímulo, do cerebelo para o córtex cerebral, mantêm o mesmo informado das mensagens simultaneamente enviadas à musculatura. Desta forma, o córtex continua envolvido como um instrumento monitor para gerar os ajustamentos necessários do movimento.

\section{Diante do exposto, pode-se considerar que:}

A-) As capacidades motoras envolvidas na habilidade psicomotora da técnica de ressuscitação cardiopulmonar são predominantemente cognitivas e motoras, envolvendo 9 (nove) capacidades perceptivomotoras e 8 (oito) capacidades de proficiência física.

B-) A técnica de ressuscitação cardiopulmonar é uma habilidade psicomotora classificada como aberta, seriada e categorizada como uma habilidade fina e global.

C-) O processo de ensino-aprendizagem da técnica de ressuscitação cardiopulmonar tem alto grau de complexidade.

\section{REFERÊNCIAS BIBLIOGRÁFICAS}

(1) Whyte D. Nursing skills. Nurs Times 1974; 70(I Suppl 1):93 6-

(2) Carvalho VL. Ensino de enfermagem e metodologia. 2 ed. Rio de Janeiro: Cultura Médica, 1979.

(3) Hardy LK. Keeping up with "Mrs. Chase" an analysis of nursing skill-learning. J Adv Nurs 1980; 5:321-7.

(4) Sheahan J. Some aspects of teaching and learning in nursing. J Adv Nurs 1980; .5:491-511.

(5) Sweeney MA. Essential skills for baccalaureate graduates: perspectives of education and service. J Nurs Adm 1980; 10:37-44.

Infante MS. Toward effective and efficient use of the clinical laboratory. Nurse Educ 1981; 6:16-9.
(6) Elliot R. Psychomotor skill acquisition in nursing students in Canada and the USA. Can. Nurs 1982; 78:25-7.

(7) Sweeney MA, Regan PA. Educators, employees, and new graduates define essential skill for baccalaureate graduates. J Nurs Adm 1982; 12:36-42.

(8)Butterfield SE. In defence of the demonstration room. Int. Nurs. Rev 1983; 30:15-20.

(9) Almeida MCP. Estudo do saber de enfermagem e sua dimensão prática. [tese] Rio de Janeiro (RJ): Escola Nacional de Saúde Pública; 1984.

(10) Friedlander MR. Avaliação das habilidades psicomotoras em enfermagem: subsídios para a construção de um instrumento. Rev Esc Enferm USP 1984; 4:72-7.

(11) Gomez GE, Gomes EA. The teaching or psychomotor skill in nursing. Nurse Educ 1984; 9:35-9.

(12) Mogan J, Thorne S. Injection giving effect of time lapse between learning and actual practice on student confidence. Nurs Pap 1985; 17:49-58.

(13) Milde FK. The function of feedback in psychomotor skill learning. West J Nurs Res 1988; 1:425-34.

(14) Miyadahira AMK. Processo de ensino-aprendizagem de habilidades psicomotoras: análise da técnica de injeção intramuscular. [tese] São Paulo (SP): Escola de Enfermagem da Universidade de São Paulo; 1990.

(15) Miyadahira AMK. Processo de ensino-aprendizagem de habilidades psicomotoras em procedimentos de emergência: avaliação e atendimento primário. [tese] São Paulo (SP). Escola de Enfermagem da Universidade de São Paulo; 1997

(16) Horta WA, Kamiyama Y, Paula NS. O ensino dos instrumentos básicos de enfermagem. Rev Esc Enferm USP 1970 4:5-20.

(17) Paim RCN. Problemas de enfermagem e a terapia centrada nas necessidades do paciente. Rio de Janeiro: União dos Cursos Cariocas; 1978.

(18) Sage GH. Introduction to motor behavior; a neurophychological approach. London: Reading, Addison-Wesley; 1971.

(19) Gagné RM. Principios essenciais de aprendizagem para o ensino. Porto Alegre: Globo; 1980.

(20) Leighbody GB, Kidd RM. Métodos para o ensino profissionalizante. São Paulo: EPU; 1980.

(21) Magill RA. Aprendizagem motora: conceitos e aplicações. São Paulo: Edgard Blucher; 1984.

(22) Enricone D. Planejamento de ensino e avaliação. 10 ed. Porto Alegre: Sagra, 1986.

(23) Singer RN, Suwanthada S. The generalizability effectiveness of learning strategy on achivement in related closed motor skills. Rev Q Exerc Sport 1986; .57:205-14. 
(25) Neary M. Project 2000 student's survival kit a return to the practical room (nursing skills laboratoty). Nurse Educ. Today, Project 1997; 17:46-52.

(26) Schimdt RA. Aprendizagem e performance motora: dos princípios à prática. São Paulo: Movimento; 1992

(27) Whyte D. Nursing skills. Nurs. Times 1974; 70(I Suppl 2):101-2.

(28) Friedlander MR. O ensino dos procedimentos básicos no laboratório de enfermagem: comparação entre dois métodos de instrução. [tese] São Paulo: Escola de Enfermagem da Universidade de São Paulo; 1984.

(29) Takiuti T, Yamashita T. Observation on the changes in the style of learning nursing technics in the course of clinical training. Kango Kyoiku 1984; 25:293-8.

(30) Hayashida M- Laboratório de Enfermagem. [dissertação] Ribeirão Preto (RP): Escola de Enfermagem de Ribeirão Preto /USP; 1995.

(31) Muramatsu CH. Processo ensino-aprendizagem da técnica de palpação e mensuração uterina: influência da prática. [dissertação] São Paulo (SP): Escola de Enfermagem da Universidade de São Paulo; 1996.

(32) Ribeiro RCN. Paramentação cirúrgica: avaliação do processo ensino-aprendizagem [dissertação] São Paulo (SP): Escola de Enfermagem da Universidade de São Paulo; 1997.

(33) Miyadahira AMK. Avaliação do processo ensinoaprendizagem de um procedimento de emergência [Apresentado no III Sociedade brasileira de atendimento integrado ao traumatizado e I Infecção no paciente critico; 1999 abr 18; São Paulo].

(34) Marteniuk RG. Information processing in motor skills. New York: Holt Rinehart and Winston; 1976.

(35) Harrow AJ. Taxionomia para o domínio psicomotor. Rio de Janeiro: Globo; 1983

(36) Tani G. Educação física escolar: fundamentos de uma abordagem desenvolvimentista. São Paulo: EPU; 1988.

(37) Magill RA. Motor learning: concepts and applications. 4 ed. United States of America: Wm C Brown Communications Inc ; 1993.

(38) Farah OGD. A ansiedade e a prática no processo ensinoaprendizagem de habilidades psicomotoras: técnica de preparo de medicação parenteral. [dissertação] São Paulo (SP): Escola de Enfermagem da Universidade de São Paulo; 1996.

(39) Real VRD. Modelo de aquisição de aquisição de destrezas motoras na enfermagem. [tese] Rio Grande do Sul (RGS): Universidade Federal de Santa Maria; 1996.

Artigo recebido em $08 / 03 / 01$

(40) Kerpharth NC. The slow learner in the classroom. 2 ed. Columbus: Charles E Merril; 1960.

\section{Artigo aprovado em 13/06/02}

(41) Fleishman EA. Structure and measurement psychomotor abilities. In: Singer RN. The psychomotor domain. Philadelphia: Lea \& Febriger; 1972. p. 78-106.
(42) Fleishman EA. Structure and measurement of physical fitness. New York: Prentice Hall, 1964.

(43) Fleishman EA. On the relationship between abilities, learning and the human performance. American Psychologist 1965; 27:1017-32.

(44) Gentile AM. A working model of skill acquisition with application to teaching. Quest 1972; 17:3-23.

(45) Gil AC. Metodologia de ensino superior. São Paulo: Atlas; 1990

(46) Fitts PM, Posner MI. Human performance. Belmont California: Brooks Cole, 1967.

(47) Singer RN. Motor learnig and human performance. 3 ed. New York: Mcmillan; 1980.

(48) Bullock Y, Boyle J, Wang MB. Physiology. 2nd ed. Pennsylvania: Harwal Publishing; 1991. p. 69-79.

(49) Ganong WE Fisiologia médica. 15 ed. Rio de Janeiro: Prentice Hall do Brasil; 1993.

(50) Tolosa ADPM, Canelas HM. Propedêutica neurológica; temas essenciais. 2ed. São Paulo: Sarvier; 1975.

(51) Ito M. Neurophysiological aspects of the cerebellar motor control system. Int J Neurol 1970; 7:162-76. 